\title{
Influence of birth weight and adult body composition on 17beta-estradiol levels in young women
}

\section{Citation}

Finstad, Sissi Espetvedt, Aina Emaus, Nancy Potischman, Emily Barret, Anne-Sofie Furberg, Peter T. Ellison, Grazyna Jasienska, and Inger Thune. 2009. Influence of birth weight and adult body composition on 17beta-estradiol levels in young women. Cancer Causes and Control 20(2): 233-242.

\section{Published Version}

http://dx.doi.org/10.1007/s10552-008-9238-2

\section{Permanent link}

http://nrs.harvard.edu/urn-3:HUL.InstRepos:2579905

\section{Terms of Use}

This article was downloaded from Harvard University's DASH repository, and is made available under the terms and conditions applicable to Open Access Policy Articles, as set forth at http:// nrs.harvard.edu/urn-3:HUL.InstRepos:dash.current.terms-of-use\#OAP

\section{Share Your Story}

The Harvard community has made this article openly available.

Please share how this access benefits you. Submit a story.

Accessibility 


\title{
Influence of birth weight and adult body composition on $17 \beta$-estradiol levels in
}

\section{young women}

\author{
Sissi Espetvedt, ${ }^{1}$ Aina Emaus, ${ }^{1}$ Nancy Potischman, ${ }^{2}$ Emily Barrett, ${ }^{3}$ Anne-Sofie Furberg, ${ }^{4}$ \\ Peter Ellison, ${ }^{5}$ Grazyna Jasienska, ${ }^{6}$ Inger Thune ${ }^{1,7}$
}

${ }^{1}$ Department of Oncology, Ullevål University Hospital, 0407 Oslo, Norway

${ }^{2}$ National Cancer Institute (NCI), EPN 4005, 6130 Executive Blvd-MSC 7344, Bethesda, MD 20892-7344, USA

${ }^{3}$ Center for Healthier Children, Families and Communities, University of California- Los Angeles 1100 Glendon Avenue, Suite 850 Los Angeles, CA 90024-6946

${ }^{4}$ Institute of Community Medicine, Faculty of Medicine, University of Tromsø, N-9037 Tromsø, Norway and Department of Microbiology and Infection control, University Hospital North Norway, N-9038 Tromsø, Norway

${ }^{5}$ Department of Anthropology, Harvard University, 11 Divinity Avenue, Cambridge, MA 02138, USA.

${ }^{6}$ Department of Epidemiology and Population Studies, Jagiellonian University, Collegium Medicum, Krakow, Poland

${ }^{7}$ The Research Council of Norway, P.O Box 2700 St.Hanshaugen, N-0131 Oslo, Norway

Key words: birth weight, adult body composition, energy balance, $17 \beta$-estradiol 
Background: Birth weight and adult body weight have independently been associated with breast cancer risk. Thus, we hypothesize that low birth weight, in combination with adult overweight, may influence premenopausal hormonal levels over an entire menstrual cycle.

Methods: Among 204 healthy women, aged 25-35 years, who participated in the Norwegian EBBA-I Study, birth weight and age at menarche, were assessed. Levels of $17 \beta$-estradiol were measured in daily saliva samples over one menstrual cycle using radioimmunoassay (RIA). Measurements of body composition; waist circumference $(\mathrm{cm})$, body mass index (BMI, $\mathrm{kg} / \mathrm{m}^{2}$ ) and total fat percentage (DEXA, \%) were assessed. Fasting blood samples were drawn, and serum concentrations of lipids and hormones determined.

Results: The participating women had mean birth weight of $3389 \mathrm{~g}$ and age at menarche 13.1 years. Women within the highest tertile of birth weight had the lowest $17 \beta$-estradiol throughout the menstrual cycle $(\mathrm{p}=0.03)$, and they tended to have a later age at menarche $(\mathrm{p}=$ 0.06). When we looked into birth weight in combination with adult attained weight, we found that women with lower birth weights, combined with excess weight during adulthood, had higher levels of free $17 \beta$-estradiol over an entire menstrual cycle compared with women with high birth weights and adult overweight. Women with birth weights $<3530$ g, who later developed excess body weight (waist $\geq 84 \mathrm{~cm}$ ), showed 33\% higher $17 \beta$-estradiol concentrations over a menstrual cycle compared with women with higher birth weights $(\geq 3530 \mathrm{~g})$ and adult excess body weight $(p=0.03)$. The association was even more pronounced in women with birth weights $<3220 \mathrm{~g}$, early age at menarche ( $<12$ years) and adult overweight.

Conclusion: Our findings support variation of premenopausal levels of $17 \beta$-estradiol in response to birth weight and energy status in adult life, suggesting that women with low birth weight in combination with adult overweight are put at risk for higher estradiol levels throughout menstrual cycles, of possible importance for breast cancer risk. 


\section{Introduction}

Several studies have observed a strong relationship between birth weight and later risk of chronic diseases, indicating that fetal conditions may influence later susceptibility to adult disease ('Forsdahl- Barker hypothesis') (1), (2), (3). Thus, women with low birth weight may have different set points for their physiological ovarian responsiveness to changes in energy balance later in life compared with women with higher birth weight.

Furthermore, the westernization of society including high levels of available energy combined with low overall energy expenditure, could provide new challenges for normal physiology and response, not only during the fetal period and early childhood, but also throughout life. It has been observed that birth size, a marker of fetal growth and intrauterine environment (4), together with later growth pattern and availability of energy, may influence later risk of chronic diseases, as for example diabetes or breast cancer (5), (6), (7), (8). However, the pattern observed is complex. Obesity during adolescence and early adult life has been observed to reduce premenopausal breast cancer risk, whereas later adult obesity, weight gain and unfavourable metabolic profiles have been observed to increase postmenopausal breast cancer risk (9).

In addition, indicators of affluent and excessive energy early in life - increased body weight during adolescence and a shift towards lower age at menarche (10), (11), (12), (13) may interact with later energy availability and body composition, thereby affecting levels of endogenous hormones. Along with the downward shift in age at menarche, increases in both body weight and waist circumference have been observed (14), (9). Age at menarche tends to occur earlier, especially in those with decreasing birth weight who gain more weight during pre-pubertal years (5), (13), (15). Interestingly, we recently observed that women who experienced early age at menarche ( $\leq 12$ years) and had a large waist to hip ratio, had $24 \%$ higher levels of estradiol over the menstrual cycle (16).

To our knowledge, little is known about the associations of variation in birth weight, adult attained body composition and premenopausal levels of $17 \beta$-estradiol over an entire menstrual cycle. In addition, the large variation in breast cancer incidence and levels of sex hormones across populations has been discussed and reflects both genetic variation (17), (18) and variation in availability of energy factors (19).

As steroid levels in saliva represent free steroid levels, rather than levels of both free and protein-bound circulating steroid, they allow for fine discrimination of functional differences in steroid signalling. Furthermore, as saliva can be readily collected from 
individuals on many occasions, it is possible to compare steroid levels across entire menstrual cycles among different women, rather than relying on one or a few timed blood samples (20).

Thus, the aim of the present study was to test whether levels of free $17 \beta$-estradiol over an entire menstrual cycle were associated with birth weight in combination with attained adult body composition. Such an interactive effect could be important for susceptibility to breast cancer.

\section{Materials and Methods}

\section{Participants and study design}

Women aged 25-35 years, living in Tromsø and surroundings during 2000-2002, were invited to participate in the Norwegian EBBA-I study by announcements both in newspapers and locally. Study participants had to meet the following criteria: self-reported regular menstruation (normal cycle length of 22-38 days within the previous 3 months), not taking hormonal contraceptives, no pregnancy or lactation over the previous 6 months, and no history of endocrinological (e.g. diabetes, hypo-/hyperthyroidism), gynecological or chronic disorders. A total of 204 women who met the inclusion criteria were subsequently enrolled into the study and came to the Department of Clinical Research, University Hospital of North Norway (UNN), at a scheduled time (21).

\section{Questionnaires}

We used a general questionnaire (self-administered and by interview) to collect information on ethnicity, education, menstruation and reproductive history, previous use of hormonal contraceptives, family history of cancer, lifetime total physical activity, smoking ['current smoker' (yes/no), 'how many cigarettes per day'] and alcohol [ 'do you drink alcohol' (yes/no), 'units of alcohol']. Trained personnel conducted interviews using recall and memory-probing aids, including a lifetime calendar. A pre-coded food diary with a photographic booklet on portion size was developed and used in order to collect 7 days of dietary data (days 3-6 and days 21-23 of the menstrual cycle, where day 1 represented the onset of menstrual bleeding) (22). Average daily intake of energy and nutrients were computed by using a food database and software system developed at the Institute for Nutrition Research, University of Oslo, Norway (23). 
Birth size, age at menarche and body composition measurements

We collected data on birth size from personal health records. Age at menarche was assessed by both questionnaire and interview using the same trained nurse during the whole study period.

Study participants made three subsequent visits to the study laboratory over the course of one menstrual period: visit 1 (days 1-4), visit 2 (midcycle) and visit 3 (days 22-25). They came in on the first possible day after onset of menstrual bleeding for clinical examinations, anthropometric measurements and provision of a fasting blood sample. All clinical procedures were conducted by trained nurses at the Department of Clinical Research, UNN, Tromsø. Anthropometric measures were taken with participants wearing lightweight clothing and no footwear: height and waist circumference were measured to the nearest half centimeter and weight to the nearest $0.1 \mathrm{~kg}$ on an electronic scale. Body Mass Index $\left(\mathrm{kg} / \mathrm{m}^{2}\right)$ was used to estimate relative weight. Waist circumference $(\mathrm{cm})$ was measured in a horizontal line $2.5 \mathrm{~cm}$ above the umbilicus.

The participants underwent a whole body scan using Dual Energy X-ray Absorptiometry or DEXA (DPX-L 2288, Lunar Radiation Corporation, Madison, WI, USA) during midcycle (days 7-12). The same trained nurse carried out the scans, and the percentage of fat tissue was estimated using Lunar software.

\section{Serum samples}

Fasting serum blood samples were drawn from an antecubital vein in the morning at all three visits. Serum concentrations of glucose and estradiol were measured in fresh sera at the Department of Clinical Chemistry, UNN, Tromsø. Serum concentrations of insulin and leptin were measured at the Hormone Laboratory, Aker University Hospital, Oslo, in serum that was stored at $-70^{\circ} \mathrm{C}$ for up to 3 years until analysis. Serum insulin and leptin were measured by RIA using kits from Linco Research Inc (St Charles, MO, USA) (21).

\section{Estradiol indices and assay procedure}

From the first day of bleeding and each day during the menstrual cycle, participants collected saliva samples at home, in the morning, according to collection protocols previously established at the Reproductive Ecology Laboratory at Harvard University, USA (24). Levels of $17 \beta$-estradiol were measured in daily saliva samples from 20 days (reverse cycle day -5 to -24) of the cycle using an ${ }^{125}$ I-labelled RIA kit (\#39100, Diagnostic Systems Laboratory, Webster, TX, USA), along with published modifications of the manufacturer's protocol (21). 
All samples were run in duplicate. All of a participant's samples were run in the same batch, with women randomly assigned to batches. Coefficients of variation were calculated based on high and low value pools (appropriate to the range of each steroid) included in each assay (21).

The sensitivity of the estradiol assay (the lowest concentration of estradiol distinguishable from 0 at the $95 \%$ level) was $4 \mathrm{pmol} / \mathrm{L}$. Average intra-assay variability (estimated from the $50 \%$ binding point of the standard curve) was $9 \%$, and the interassay variability ranged from $23 \%$ for low values $(15 \mathrm{pmol} / \mathrm{L})$ to $13 \%$ for high values $(50 \mathrm{pmol} / \mathrm{L})$. Before statistical analysis, all cycles were aligned to the day of ovulation following published methods (21), based on the identification of the estradiol drop at the midcycle (day 0), which provides a reasonable estimate of the day of ovulation. The estradiol values for 20 consecutive days from each cycle, aligned on day 0 , were used in data analyses (day -10 to +9). Satisfactory identification of the midcycle estradiol drop could not be made for 14 women and so their cycles were not aligned. However, in order to study the total variation in estradiol concentration throughout an entire menstrual cycle and as anovulatory cycles are associated with low estradiol exposure, all cycles; both anovulatory and ovulatory cycles, were included in this study.

\section{Statistical analysis}

The associations between birth weight, adult attained body composition and $17 \beta$-estradiol levels throughout a menstrual cycle were studied using linear regression analysis and linear mixed models for repeated measures (SAS statistical package version 9.1).

To study in detail the association between birth weight and $17 \beta$-estradiol levels, the study population was divided into birth weight tertiles: $<3220 \mathrm{~g}, \geq 3220$ to $3530 \mathrm{~g}$ and $\geq 3530 \mathrm{~g}$. These groups of women with different birth weights were then compared with regard to selected characteristics. We used one-way analysis of variance for continuous variables and $\chi^{2}$ tests for categorical variables to test for differences in means and frequencies of selected characteristics across tertiles of birth weight.

Linear regression models were used to study the associations of average salivary $17 \beta-$ estradiol concentration, birth weight, and measures of adult attained body composition, serum insulin and leptin. Potential confounding factors were tested and adjusted for when appropriate. Based on biological plausibility, covariates such as age, smoking, physical activity, age at menarche, energy intake, alcohol, previous use of hormonal contraceptives, 
age at first birth and number of children were tested in the models. Age, smoking, physical activity and age at menarche were included in the final models due to proper model-data fit.

We evaluated possible interactions between measures of attained adult body composition and serum insulin and leptin and birth weight (tertiles) by including multiplicative interaction tests in the models.

To study whether variation in adult excess body weight and fat distribution modified the association between birth weight and salivary concentrations of $17 \beta$-estradiol, waist circumference were dichotomized at the 75 th percentile $(\geq 84 \mathrm{~cm})$. We used linear mixed models for repeated measures to study variations in salivary $17 \beta$-estradiol concentrations over the entire menstrual cycle across different subgroups of women defined by birth weight (tertiles) and adult body composition (75th percentile); age was included in the models. As the multivariate analyses only gave minor changes of our age-adjusted estimates, both in relation to linear regression models and in relation to linear mixed models for repeated measures, only age adjusted results are presented in figures using mixed models for repeated measures. Different co-variance structures were explored, and the results are presented using heterogeneous Toeplitz. Dunnett's method was used for multiple comparisons.

Measurements of $17 \beta$-estradiol at the start and the finish of the cycles had higher coefficients of variation and higher rates of missing data as a result of variation in cycle length; therefore we included $17 \beta$-estradiol measurements from cycle day -10 to +9 in the linear mixed models. Results were considered statistically significant when the two-sided $p$ value was $<0.05$.

\section{Ethical considerations}

All the participating women signed an informed consent form. The study protocol was reviewed and approved by the Regional Committee for Medical Research Ethics NorthNorway and the Norwegian Data Inspectorate.

\section{Results}

The 204 participating women had mean age 30.7 years, mean birth weight $3389 \mathrm{~g}$ and selfreported mean age at menarche 13.1 years. Mean BMI was $24.4 \mathrm{~kg} / \mathrm{m}^{2}$, mean waist circumference $79.5 \mathrm{~cm}$ and mean total fat percentage $34.1 \%$. When dividing the women into tertiles of birth weight $(<3220 \mathrm{~g}, \geq 3220$ to $3530 \mathrm{~g}$ and $\geq 3530 \mathrm{~g}$ ), women in the highest tertile of birth weight had the lowest overall average salivary and thus free $17 \beta$-estradiol 
concentrations $(p=0.03)$, and tended to have a later age at menarche $(p=0.06)$ (Table 1$)$. In addition, women with the lowest birth weight had significantly higher serum insulin levels ( $p$ $=0.02$ ) compared with women with higher birth weights.

We also studied the average $17 \beta$-estradiol by cycle day over the entire menstrual cycle across birth weight tertiles. Women who reported the highest birth weight ( $\geq 3530 \mathrm{~g})$ tended to have the lowest levels of daily free $17 \beta$-estradiol over the entire menstrual cycle (Figure 1a).

No clear pattern was observed between $17 \beta$-estradiol by cycle day over the entire menstrual cycle by tertiles of adult attained waist circumference (Figure 1b).

We then studied the changes in overall average salivary $17 \beta$-estradiol concentration by 1 standard deviation (SD) change in explanatory variables for each tertile of birth weight. Among women in the two lowest tertiles of birth weight $(<3530 \mathrm{~g})$, we observed a pattern of positive association between measures of adult attained body composition and serum leptin and insulin levels and mean overall $17 \beta$-estradiol concentration in both age-adjusted models and models adjusted for potential confounders (smoking, physical activity and age at menarche). For each $3.8 \mathrm{~kg} / \mathrm{m}^{2}$ (1 SD) increase in BMI, the overall adjusted level of $17 \beta$ estradiol in the lowest birth weight tertile increased by $2.74 \mathrm{pmol} / \mathrm{L}(95 \% \mathrm{CI}, 0.63,4.85)$, and in the middle birth weight tertile by $2.84 \mathrm{pmol} / \mathrm{L}(95 \% \mathrm{CI}, 0.56,5.11)$, equivalent to a $15.3 \%$ change in mean average concentration of $17 \beta$-estradiol in the lowest birth weight tertile and a $15.9 \%$ change in the middle birth weight tertile, respectively. In contrast, among women in the highest tertile of birth weight $(\geq 3530 \mathrm{~g})$, the level of $17 \beta$-estradiol did not vary with changes in adult body composition and serum hormones (Table 2).

When we examined changes in waist circumference by tertiles of birth weight, we observed that for each $9.8 \mathrm{~cm}$ (1 SD) increase in waist circumference, the overall adjusted level of $17 \beta$-estradiol in the lowest birth weight tertile tended to increase. Among women in the middle birth weight tertile each 1 SD increase in waist circumference was associated with an increase of $3.05 \mathrm{pmol} / \mathrm{L}$ (95\% CI, 0.53,5.57) in overall average $17 \beta$-estradiol levels, which equals a $17.0 \%$ change in overall average $17 \beta$-estradiol concentration. The results were even more pronounced when we performed the analyses by birth weight in quartiles (data not shown).

To study in detail whether age at menarche influenced our result, we performed analysis stratified by age at menarche. Among women with low birth weight $(<3220 \mathrm{~g})$, the relationship between measures of excess adult waist circumference and overall $17 \beta$-estradiol 
concentrations was strongest in the subgroup of those having an age at menarche $<12$ years. For each $9.8 \mathrm{~cm}(1 \mathrm{SD})$ increase in waist, overall average $17 \beta$-estradiol concentration increased by $3.9 \mathrm{pmol} / \mathrm{L}$, which equals a $21.8 \%$ change in mean overall $17 \beta$-estradiol levels among women in the lowest birth weight tertile ( $p=0.03$; not presented in tables).

In our study we observed a significant relationship between birth weight and serum insulin levels. When we examined birth weight in relation to adult overweight, the results became even clearer. Women of low birth weight $(<3220 \mathrm{~g})$ who later became overweight (as defined by a waist circumference $\geq 84 \mathrm{~cm})$ had twice as high insulin levels $(145.4 \mathrm{pmol} / \mathrm{L})$ than women of low birth weight and adult waist circumference $<84 \mathrm{~cm}(77.7 \mathrm{pmol} / \mathrm{l} ; p=$ 0.0018; data not shown in tables).

To study the differences in daily salivary $17 \beta$-estradiol concentrations over an entire menstrual cycle between subgroups of women characterized by birth weight and adult attained waist, we used a linear mixed model for repeated measures (Figures 2a-c). We observed that birth weight combined with adult waist circumference showed a clear association with levels of premenopausal $17 \beta$-estradiol throughout an entire menstrual cycle. Women with birth weights $<3530 \mathrm{~g}$ who later developed excess body weight (waist $\geq 84 \mathrm{~cm}$ ) showed higher $17 \beta$-estradiol concentrations over the menstrual cycle compared with women of higher birth weights ( $\geq 3530 \mathrm{~g}$ ) and adult excess body weight ( $p=0.03$; Figure 2a). These results equal a change in mean overall concentration of $17 \beta$-estradiol for the total group of 33 $\%$. Comparable results were also observed among women with an early age at menarche compared to later age at menarche; among women of lower birth weight $(<3530 \mathrm{~g})$ and adult excess waist, those with an early age at menarche ( $<12$ years) had higher levels of $17 \beta$ estradiol over their menstrual cycle compared with those who had a later age at menarche $(p=$ 0.07; Figure $2 \mathrm{~b})$. Women with lower birth weights $(<3530 \mathrm{~g})$, early age at menarche $(<12$ years) and adult overweight also tended to have higher insulin levels than those who were older at menarche ( $\geq 12$ years), although the difference was not significant (data not presented). Among those in the lowest birth weight tertile $(<3220 \mathrm{~g})$ who later had a larger waist circumference ( $\geq 84 \mathrm{~cm}$, highest quartile), there was a trend for those with early age at menarche $(<12$ years $)$ to have even higher levels of $17 \beta$-estradiol over a whole menstrual cycle compared with those with later age at menarche ( $p=0.09$; Figure $2 c)$.

\section{Discussion}


In our study of young healthy women with regular menstrual cycles we found that low birth weight $(<3530 \mathrm{~g})$ combined with large attained adult waist $(\geq 84 \mathrm{~cm})$ resulted in a $33 \%$ increase in free $17 \beta$-estradiol levels over an entire menstrual cycle compared with women of higher birth weight and the same adult waist circumference. This association was even more pronounced among women of even lower birth weights, $<3220 \mathrm{~g}$, combined with early age at menarche ( $<12$ years).

Little is known about the interrelationship of birth weight, adult attained body composition and $17 \beta$-estradiol levels over an entire menstrual cycle. Therefore, one could ask whether these results can be explained by plausible biological mechanisms, observations from other studies or by bias and/or confounding. Interestingly, several studies have observed that perinatal factors, including birth weight, may influence later susceptibility of chronic diseases including breast cancer (25), (26), (5), (27), (28). Moreover, the association related to breast cancer has been complicated by contradictory results (29), (30) because some have observed a protective effect of low birth weight (25), whereas others have not (31). We have previously hypothesized that birth size may influence later responsiveness of ovarian function and have shown that, among adult women ovarian response to physical activity depends on their size at birth (32). Women of low birth weights may have a different set point for their physiological responsiveness than women with higher birth weights. Barker has proposed that fetal tissues respond to the intrauterine environment by permanently altering their structure and function (2). Such a mechanism may explain associations between low birth weight and increased risk of chronic diseases (33), including breast cancer risk.

Other studies have shown a clear interrelationship of low birth weight, later overweight and susceptibility of chronic diseases (34). One biological explanation of this could be that individuals programmed for poor early nutrient intake would be put at risk if their food intake was subsequently increased to a level inappropriate for their programming(35).

Lissner et al. (36) furthermore found that birth weight was inversely related to serum leptin levels in adulthood, and that leptin can stimulate mitogenic and angiogenic processes in peripheral organs. Data obtained in cell and animal models and analyses of human breast cancer biopsies suggest involvement in breast cancer etiology (37). In our study we observed that serum leptin levels tended to be lower among women in the highest tertile of birth weight, although this result was not significant (Table 1).

There is a growing recognition that breast cancer may be promoted by hyperinsulinemia and insulin resistance, which may favour a metabolic environment 
promoting tumor growth (38). Additionally, insulin resistance and elevated serum steroid levels often coexist because of insulin up-regulation of ovarian steroid secretion (39). Low birth weight, thinness at 2 years of age and an increase in BMI after the age of 2 have been observed to be associated with development of insulin resistance in later life (40), (41), (42) underlining the importance of birth size as a risk factor for insulin resistance and other chronic diseases. Our study supports these findings by showing a significant relationship ( $p=$ 0.02 ) between tertiles of birth weight and serum insulin levels. The results became even clearer when we looked into birth weight in relation to being overweight as an adult. Women of low birth weight $(<3220 \mathrm{~g})$ who later became overweight (with a waist circumference $\geq 84 \mathrm{~cm}$ ) had significantly higher insulin levels, compared with those of low birth weight and adult waist circumference $<84 \mathrm{~cm}$. It is suggested that metabolic markers of high breast cancer risk include higher serum concentrations of insulin and IGF1 and also excessive abdominal fat accumulation (43).

Mechanisms during gestation, such as increasing growth factor exposure and availability of energy influencing birth weight, may also influence later responsiveness to other factors. However, effect modification throughout childhood and adult life may interact and, among others, levels of $17 \beta$-estradiol over a menstrual cycle may vary.

Several biological mechanisms and observation form different studies support a relationship between birth weight, childhood and adult weight gain and $17 \beta$-estradiol. Some studies have observed that childhood obesity may accelerate age at menarche, especially in those of low birth weight (5),(13),(44),(45), and early age at menarche is a known risk factor for breast cancer development. In our study we observed that women of birth weight $<3530 \mathrm{~g}$ tended to have an earlier age at menarche than those in the highest birth weight tertile, $\geq 3530$ g. A Swedish study suggested that girls who at birth were relatively small for gestational age had higher childhood growth velocities than other girls (46). These results indicate that there may be compensation for those with fetal growth restriction through higher growth rate after birth. It is also possible that such compensatory growth could be mediated by increased levels of adrenal steroids and hyperinsulinemia in girls who were relatively small at birth (47), (40). Our observation that women with low birth weight and adult obesity tend to have higher levels of both insulin and $17 \beta$-estradiol over a menstrual cycle supports these findings. On the other hand, higher birth weight together with low pre-pubertal body weight have been observed to result in later age at menarche and lower estradiol concentrations (48). 
Ovarian steroids are thought to increase the rate of breast cancer growth mainly by stimulating the mitotic activity of breast epithelial cells both directly and through interaction with growth hormones (49). One implication of the accumulating evidence on the importance of ovulatory menstrual cycles is that any factor that modifies menstrual cycle patterns and reduces the frequency of ovulation may reduce a woman's lifetime risk of developing breast cancer (50). Consequently, reducing lifetime estrogen levels might be the most important step in lowering breast cancer in women.

The use of just one clinical research department at a university hospital with one specially trained nurse enhanced the quality of our data. It allowed us to sample all clinical variables within the same narrow frame of the cycle for each participant, using uniform procedures. To limit any potential influence of variation in daylight, women did not participate during those months with markedly less daylight (December and January).

The present population consists of only 204 women which limit the possibility to perform subgroup analyses. Moreover, it also underlines the necessity to interpret our results with cautiousness and the need for further studies among other ethnic groups. However, the population is very homogenous which may strengthen the interpretation in smaller groups. Taking daily saliva samples allowed estimation of daily estradiol concentrations over one menstrual cycle, enabling precise and reliable assessment of interindividual variations in hormonal levels. We used well-developed and validated methods and assays to characterize the women's exposure to free, biologically active, ovarian steroids and the comparison of levels by aligned cycle days (24). This study had the benefit of collecting samples every day over an entire menstrual cycle, rather than only on selected days within a cycle (51). In addition, salivary levels of estradiol were shown to be quite stable within participants over time (52).

Age at menarche was recalled retrospectively, and misclassification is therefore likely. Several studies, however, have found that age at menarche are recalled with high reliability (53). In multivariate and stratified analyses we adjusted for potential confounders as age, smoking, physical activity and age at menarche. They were included in the final model without any large changes in estimates.

\section{Conclusion}


Our main findings support that lower birth weight, combined with large waist circumference during adulthood, is associated with increased levels of $17 \beta$-estradiol over one menstrual cycle, especially among women with early age at menarche ( $<12$ years). These women also had higher levels of insulin. This supports the hypothesis that women of lower birth weight may have a different set point for their physiological responsiveness than women of higher birth weight; this may, in turn, influence levels of reproductive hormones such as $17 \beta-$ estradiol. Our findings support the hypothesis that conditions during fetal, childhood and adult life may in combination influence later breast cancer risk.

\section{Acknowledgements}

We acknowledge each woman who participated in the Norwegian EBBA-I study, our nurse Gunn Knudsen, Anna Kirsti Jenssen and Sissel Andersen. The study was supported by a grant from the Norwegian Cancer Society (49 258, 05087); Foundation for the Norwegian Health and Rehabilitation Organizations (59010-2000/2001/2002); Aakre Foundation (5695-2000, 5754-2002) and Health Region East.

Reference List 
(1) Forsdahl A. Observations throwing light on the high mortality in the county of Finnmark. Is the high mortality today a late effect of very poor living conditions in childhood and adolescence? 1973. Int J Epidemiol 2002 Apr;31(2):302-8.

(2) Barker DJ. In utero programming of chronic disease. Clin Sci (Lond) 1998 Aug;95(2):115-28.

(3) Lau C, Rogers JM. Embryonic and fetal programming of physiological disorders in adulthood. Birth Defects Res C Embryo Today 2004 Dec;72(4):300-12.

(4) Trichopoulos D. Is breast cancer initiated in utero? Epidemiology 1990 Mar;1(2):95-6.

(5) Romundstad PR, Vatten LJ, Nilsen TI, Holmen TL, Hsieh CC, Trichopoulos D, et al. Birth size in relation to age at menarche and adolescent body size: implications for breast cancer risk. Int J Cancer 2003 Jun 20;105(3):400-3.

(6) Ozanne SE, Jensen CB, Tingey KJ, Martin-Gronert MS, Grunnet L, Brons C, et al. Decreased protein levels of key insulin signalling molecules in adipose tissue from young men with a low birthweight: potential link to increased risk of diabetes? Diabetologia 2006 Dec;49(12):2993-9.

(7) McCormack VA, dos SS, I, Koupil I, Leon DA, Lithell HO. Birth characteristics and adult cancer incidence: Swedish cohort of over 11,000 men and women. Int J Cancer 2005 Jul 1;115(4):611-7.

(8) dos SS, I, De Stavola BL, Hardy RJ, Kuh DJ, McCormack VA, Wadsworth ME. Is the association of birth weight with premenopausal breast cancer risk mediated through childhood growth? Br J Cancer 2004 Aug 2;91(3):519-24. 
(9) IARC. IARC Handbook of Cancer Prevention. Weight control and physical activity. Lyon, International Agency for Research on Cancer (IARC), 2002. 2007. Ref Type: Generic

(10) Kirchengast S, Gruber D, Sator M, Huber J. Impact of the age at menarche on adult body composition in healthy pre- and postmenopausal women. Am J Phys Anthropol 1998 Jan;105(1):9-20.

(11) Adair LS. Size at birth predicts age at menarche. Pediatrics 2001 Apr;107(4):E59.

(12) Chavarro JE, Peterson KE, Sobol AM, Wiecha JL, Gortmaker SL. Effects of a schoolbased obesity-prevention intervention on menarche (United States). Cancer Causes Control 2005 Dec;16(10):1245-52.

(13) Dunger DB, Ahmed ML, Ong KK. Early and late weight gain and the timing of puberty. Mol Cell Endocrinol 2006 Jul 25;254-255:140-5.

(14) Ballard-Barbash R. Anthropometry and breast cancer. Body size--a moving target. Cancer 1994 Aug 1;74(3 Suppl):1090-100.

(15) van Weissenbruch MM, Delemarre-van de Waal HA. Early influences on the tempo of puberty. Horm Res 2006;65 Suppl 3:105-11.

(16) Emaus. 17 beta-estradiol in relation to age at menarche and adult obesity in premenopausal women, in press CEBP. 2007.

Ref Type: Generic

(17) Bernstein L. Epidemiology of endocrine-related risk factors for breast cancer. J Mammary Gland Biol Neoplasia 2002 Jan;7(1):3-15. 
(18) King SE, Schottenfeld D. The "epidemic" of breast cancer in the U.S.--determining the factors. Oncology (Williston Park) 1996 Apr;10(4):453-62.

(19) Jasienska G, Thune I. Lifestyle, hormones, and risk of breast cancer. BMJ 2001 Mar $10 ; 322(7286): 586-7$.

(20) Jasienska G, Kapiszewska M, Ellison PT, Kalemba-Drozdz M, Nenko I, Thune I, et al. CYP17 genotypes differ in salivary 17-beta estradiol levels: a study based on hormonal profiles from entire menstrual cycles. Cancer Epidemiol Biomarkers Prev 2006 Nov;15(11):2131-5.

(21) Furberg AS, Jasienska G, Bjurstam N, Torjesen PA, Emaus A, Lipson SF, et al. Metabolic and hormonal profiles: HDL cholesterol as a plausible biomarker of breast cancer risk. The Norwegian EBBA Study. Cancer Epidemiol Biomarkers Prev 2005 Jan;14(1):33-40.

(22) Lillegaard IT, Overby NC, Andersen LF. Can children and adolescents use photographs of food to estimate portion sizes? Eur J Clin Nutr 2005 Apr;59(4):611-7.

(23) Andersen LF. Is there any difference in what children are eating during weekends and the rest of the week? 2003.

Ref Type: Generic

(24) Lipson SF, Ellison PT. Comparison of salivary steroid profiles in naturally occurring conception and non-conception cycles. Hum Reprod 1996 Oct;11(10):2090-6.

(25) Michels KB, Trichopoulos D, Robins JM, Rosner BA, Manson JE, Hunter DJ, et al. Birthweight as a risk factor for breast cancer. Lancet 1996 Dec 7;348(9041):1542-6. 
(26) Ahlgren M, Sorensen T, Wohlfahrt J, Haflidadottir A, Holst C, Melbye M. Birth weight and risk of breast cancer in a cohort of 106,504 women. Int J Cancer 2003 Dec 20;107(6):997-1000.

(27) Vatten LJ, Nilsen TI, Tretli S, Trichopoulos D, Romundstad PR. Size at birth and risk of breast cancer: prospective population-based study. Int J Cancer 2005 Apr $10 ; 114(3): 461-4$

(28) Mellemkjaer L, Olsen ML, Sorensen HT, Thulstrup AM, Olsen J, Olsen JH. Birth weight and risk of early-onset breast cancer (Denmark). Cancer Causes Control 2003 Feb;14(1):61-4.

(29) Troisi R, Hatch EE, Titus-Ernstoff L, Palmer JR, Hyer M, Strohsnitter WC, et al. Birth weight and breast cancer risk. Br J Cancer 2006 Jun 5;94(11):1734-7.

(30) Michels KB, Willett WC. Breast cancer--early life matters. N Engl J Med 2004 Oct $14 ; 351(16): 1679-81$.

(31) Troisi R, Hatch EE, Titus-Ernstoff L, Palmer JR, Hyer M, Strohsnitter WC, et al. Birth weight and breast cancer risk. Br J Cancer 2006 Jun 5;94(11):1734-7.

(32) Jasienska G, Thune I, Ellison PT. Fatness at birth predicts adult susceptibility to ovarian suppression: an empirical test of the Predictive Adaptive Response hypothesis. Proc Natl Acad Sci U S A 2006 Aug 22;103(34):12759-62.

(33) Leong NM, Mignone LI, Newcomb PA, Titus-Ernstoff L, Baron JA, Trentham-Dietz A, et al. Early life risk factors in cancer: the relation of birth weight to adult obesity. Int J Cancer 2003 Mar 1;103(6):789-91. 
(34) Barker DJ, Forsen T, Eriksson JG, Osmond C. Growth and living conditions in childhood and hypertension in adult life: a longitudinal study. J Hypertens 2002 Oct;20(10):1951-6.

(35) Lucas A, Fewtrell MS, Cole TJ. Fetal origins of adult disease-the hypothesis revisited. BMJ 1999 Jul 24;319(7204):245-9.

(36) Lissner L, Karlsson C, Lindroos AK, Sjostrom L, Carlsson B, Carlsson L, et al. Birth weight, adulthood BMI, and subsequent weight gain in relation to leptin levels in Swedish women. Obes Res 1999 Mar;7(2):150-4.

(37) Surmacz E. Obesity hormone leptin: a new target in breast cancer? Breast Cancer Res 2007 Jan 26;9(1):301.

(38) Sieri S, Pala V, Brighenti F, Pellegrini N, Muti P, Micheli A, et al. Dietary glycemic index, glycemic load, and the risk of breast cancer in an Italian prospective cohort study. Am J Clin Nutr 2007 Oct;86(4):1160-6.

(39) Barbieri RL, Smith S, Ryan KJ. The role of hyperinsulinemia in the pathogenesis of ovarian hyperandrogenism. Fertil Steril 1988 Aug;50(2):197-212.

(40) Barker DJ. The developmental origins of insulin resistance. Horm Res 2005;64 Suppl $3: 2-7$

(41) Phillips DI, Barker DJ, Hales CN, Hirst S, Osmond C. Thinness at birth and insulin resistance in adult life. Diabetologia 1994 Feb;37(2):150-4.

(42) Jeffreys M, Lawlor DA, Galobardes B, McCarron P, Kinra S, Ebrahim S, et al. Lifecourse weight patterns and adult-onset diabetes: the Glasgow Alumni and British Women's Heart and Health studies. Int J Obes (Lond) 2006 Mar;30(3):507-12. 
(43) Stoll BA. Adiposity as a risk determinant for postmenopausal breast cancer. Int J Obes Relat Metab Disord 2000 May;24(5):527-33.

(44) Newby PK, Dickman PW, Adami HO, Wolk A. Early anthropometric measures and reproductive factors as predictors of body mass index and obesity among older women. Int J Obes (Lond) 2005 Sep;29(9):1084-92.

(45) Cooper C, Kuh D, Egger P, Wadsworth M, Barker D. Childhood growth and age at menarche. Br J Obstet Gynaecol 1996 Aug;103(8):814-7.

(46) Persson I, Ahlsson F, Ewald U, Tuvemo T, Qingyuan M, von RD, et al. Influence of perinatal factors on the onset of puberty in boys and girls: implications for interpretation of link with risk of long term diseases. Am J Epidemiol 1999 Oct $1 ; 150(7): 747-55$.

(47) de ZF, Ibanez L. Novel insights into the endocrine-metabolic and reproductive consequences of prenatal growth restraint in girls. Girls-born-small become womenborn-small. Verh K Acad Geneeskd Belg 2004;66(5-6):353-82.

(48) Bernstein L, Pike MC, Ross RK, Henderson BE. Age at menarche and estrogen concentrations of adult women. Cancer Causes Control 1991 Jul;2(4):221-5.

(49) McPherson K, Steel CM, Dixon JM. ABC of breast diseases. Breast cancerepidemiology, risk factors, and genetics. BMJ 2000 Sep 9;321(7261):624-8.

(50) Bernstein L, Henderson BE, Hanisch R, Sullivan-Halley J, Ross RK. Physical exercise and reduced risk of breast cancer in young women. J Natl Cancer Inst 1994 Sep 21;86(18):1403-8. 
(51) Jasienska G, Jasienski M. Interpopulation, interindividual, intercycle, and intracycle natural variation in progesterone levels: a quantitative assessment and implications for population studies. Am J Hum Biol 2008 Jan;20(1):35-42.

(52) Ellison PT, Lipson SF. Salivary estradiol--a viable alternative? Fertil Steril 1999 Nov;72(5):951-2.

(53) Must A, Phillips SM, Naumova EN, Blum M, Harris S, wson-Hughes B, et al. Recall of early menstrual history and menarcheal body size: after 30 years, how well do women remember? Am J Epidemiol 2002 Apr 1;155(7):672-9. 
Table 1 Characteristics of the study population in tertiles of birth weight, means (SD)*: The Norwegian EBBA-Study $\left(N=204^{\mathrm{a}}\right)$

\section{Birth weight}

\begin{tabular}{|c|c|c|c|c|}
\hline Study characteristics & $\begin{array}{l}<3220 \mathrm{~g} \\
(n=68)\end{array}$ & $\begin{array}{c}\geq 3220-3530 \mathrm{~g} \\
\quad(n=68)\end{array}$ & $\begin{array}{c}\geq 3530 \mathrm{~g} \\
(n=68)\end{array}$ & $\begin{array}{l}p \text { value }^{b} \\
\text { (trend) }\end{array}$ \\
\hline Age (years) & $30.8(3.2)$ & $30.8(2.9)$ & $30.6(3.2)$ & 0.71 \\
\hline Years of schooling & $15.7(3.2)$ & $16.2(2.8)$ & $16.3(3.1)$ & 0.22 \\
\hline Ethnic minority, Sami (\%) & 10.3 & 7.4 & 5.9 & 0.62 \\
\hline \multicolumn{5}{|l|}{ Anthropometric measurements } \\
\hline $\operatorname{BMI}\left(\mathrm{kg} / \mathrm{m}^{2}\right)$ & $23.9(3.6)$ & $25.5(4.1)$ & $23.9(3.4)$ & 0.96 \\
\hline Waist circumference $(\mathrm{cm})$ & $78.2(10.3)$ & $81.4(9.9)$ & $79.0(9.1)$ & 0.60 \\
\hline Total fat $(\%)$ & $33.8(8.3)$ & $36.1(7.1)$ & $32.5(7.1)$ & 0.35 \\
\hline \multicolumn{5}{|l|}{ Saliva hormone concentrations (pmol/L) } \\
\hline Overall $17 \beta$-estradiol & $18.8(8.3)$ & $19.5(10.2)$ & $15.5(7.3)$ & 0.03 \\
\hline \multicolumn{5}{|l|}{ Serum hormone concentrations $\mathrm{s}^{\mathrm{c}}$} \\
\hline Serum estradiol (nmol/L) & $0.14(0.1)$ & $0.15(0.1)$ & $0.15(0.1)$ & 0.50 \\
\hline Serum leptin $(\mathrm{pmol} / \mathrm{L})$ & $826.8(561.7)$ & $968.8(571.7)$ & $772.8(544.9)$ & 0.58 \\
\hline Serum glucose $(\mathrm{mmol} / \mathrm{L})$ & $5.0(0.6)$ & $5.1(0.5)$ & $5.0(0.6)$ & 0.59 \\
\hline Serum insulin $(\mathrm{pmol} / \mathrm{L})$ & $95.6(80.8)$ & $90.1(53.0)$ & $71.5(30.9)$ & 0.02 \\
\hline \multicolumn{5}{|l|}{ Menstrual and reproductive characteristics } \\
\hline Menarche (years) & $12.96(1.3)$ & $12.98(1.3)$ & $13.40(1.5)$ & 0.06 \\
\hline Age at first birth (years) ${ }^{d}$ & $23.0(4.2)$ & $25.7(3.8)$ & $24.6(3.2)$ & 0.10 \\
\hline Percentage women with children & 45.6 & 50.0 & 48.5 & 0.73 \\
\hline Cycle length (days) & $28.3(3.0)$ & $28.7(3.3)$ & $27.8(3.1)$ & 0.36 \\
\hline \multicolumn{5}{|l|}{ Previous use of hormonal contraceptives (\%) } \\
\hline & 80.9 & 88.1 & 82.4 & 0.82 \\
\hline Energy intake (kJ/day) & $7968(1452)$ & $7976(2093)$ & $8335(2088)$ & 0.26 \\
\hline Leisure time (MET h/week) & $51.8(36.8)$ & $51.8(33.6)$ & $53.7(38.2)$ & 0.76 \\
\hline Alcohol units per week among users $(\mathrm{n}=190)$ & $2.8(3.4)$ & $3.3(3.6)$ & $3.2(3.2)$ & 0.47 \\
\hline Current smokers $(\%)$ & 20.6 & 14.7 & 30.9 & 0.15 \\
\hline
\end{tabular}

*SD, standard deviation.

${ }^{\text {a }}$ Numbers of participants may vary as a result of missing information for certain variables.

${ }^{\mathrm{b}}$ One-way analysis of variance or $\chi^{2}$ test.

${ }^{\mathrm{c}}$ Blood sampling first visit (days $1-5$ ).

${ }^{\mathrm{d}}$ For those who have children, $n=98$, in each group: $31-34-33$. 
Table 2 Estimated changes ${ }^{*}$ in mean salivary $17 \beta$-estradiol concentrations (pmol/L) with $95 \%$ CI by 1 standard deviation (SD) increase in explanatory variable by tertiles of birth weight $(n=204)^{\mathrm{a}}$

\section{Change in $17 \beta$-estradiol levels $(\mathrm{pmol} / \mathrm{L})$}

\begin{tabular}{|c|c|c|c|c|c|c|c|c|}
\hline \multirow[t]{3}{*}{ Variable } & \multirow[t]{3}{*}{ Mean } & \multirow[t]{3}{*}{ (SD) } & \multicolumn{6}{|c|}{ Birth weight } \\
\hline & & & \multicolumn{2}{|c|}{$\begin{array}{l}<3220 \mathrm{~g} \\
(n=68)\end{array}$} & \multicolumn{2}{|c|}{$\begin{array}{c}\geq 3220 \mathrm{~g},<3530 \mathrm{~g} \\
(n=68)\end{array}$} & \multicolumn{2}{|c|}{$\begin{array}{r}\geq 3530 \mathrm{~g} \\
(n=68)\end{array}$} \\
\hline & & & Age adjusted & Adjusted $^{b}$ & Age adjusted & Adjusted $^{b}$ & Age adjusted & Adjusted $^{b}$ \\
\hline BMI $\left(\mathrm{kg} / \mathrm{m}^{2}\right)$ & 24.4 & $(3.8)$ & $2.33(0.23,4.42)$ & $2.74(0.63,4.85)$ & $2.97(0.82,5.12)$ & $2.84(0.56,5.11)$ & $0.11(-1.88,2.09)$ & $0.17(-1.72,2.07)$ \\
\hline Waist (cm) & 79.5 & $(9.8)$ & $1.17(-0.79,3.13)$ & $1.67(-0.31,3.66)$ & $3.20(0.85,5.55)$ & $3.05(0.53,5.57)$ & $-0.16(-2.08,1.76)$ & $-0.28(-2.12,1.55)$ \\
\hline Total fat (\%) & 34.1 & (7.6) & $1.02(-0.85,2.90)$ & $1.69(-0.26,3.64)$ & $2.41(-0.22,5.03)$ & $2.25(-0.50,5.01)$ & $0.24(-1.68,2.15)$ & $-0.09(-2.01,1.83)$ \\
\hline Leptin (pmol/L) & 856.1 & $(562.9)$ & $1.93(-0.08,3.94)$ & $2.93(0.87,4.98)$ & $1.43(-1.00,3.85)$ & $1.24(-1.27,3.75)$ & $-0.26(-2.1,1.57)$ & $-0.12(-1.88,1.64)$ \\
\hline Insulin $(\mathrm{pmol} / \mathrm{L})$ & 85.7 & $(59.2)$ & $0.07(-1.44,1.57)$ & $0.15(-1.42,1.73)$ & $3.60(0.96,6.24)$ & $3.75(1.00,6.50)$ & $-0.56(-3,95,2,83)$ & $-0.22(-3.54,3.09)$ \\
\hline
\end{tabular}

*Linear regression analyses. Regression coefficient and 95\% confidence interval (CI).

${ }^{a}$ Number may vary as a result of missing serum values.

${ }^{\mathrm{b}}$ Adjusted for age, leisure time physical activity, number of cigarettes and age at menarche.

$95 \% \mathrm{CI}=95 \%$ confidence interval; $\mathrm{SD}$, standard deviation 


\section{Figure 1}

a) Average $17 \beta$-estradiol by cycle day over the entire menstrual cycle across tertiles of birth weight $(\mathrm{BW}),(<3220 \mathrm{~g}, \geq 3220 \mathrm{~g}$ and $<3530 \mathrm{~g}, \geq 3530 \mathrm{~g})$.

b) Average $17 \beta$-estradiol by cycle day over the entire menstrual cycle by tertiles of adult attained waist circumference (Waist), $(<74 \mathrm{~cm}, \geq 74$ and $<82 \mathrm{~cm}, \geq 82 \mathrm{~cm}$ ).

\section{Figure 2}

a) Age-adjusted salivary $17 \beta$-estradiol concentrations by cycle day in women categorized by anthropometric measurements, highest compared with lower birth weight (BW) tertiles combined with being overweight in adulthood (waist $\geq 84 \mathrm{~cm}$ ).

b) Age-adjusted salivary $17 \beta$-estradiol concentrations by cycle day in women categorized by birth weight $(\mathrm{BW})<3530 \mathrm{~g}$, adult obesity (waist $\geq 84 \mathrm{~cm}$ ) and age at menarche.

c) Age-adjusted salivary $17 \beta$-estradiol concentrations by cycle day in women categorized by birth weight $(\mathrm{BW})<3220 \mathrm{~g}$ (lowest tertile), adult obesity (waist $\geq 84 \mathrm{~cm}$ ) and age at menarche. 
Figure1a

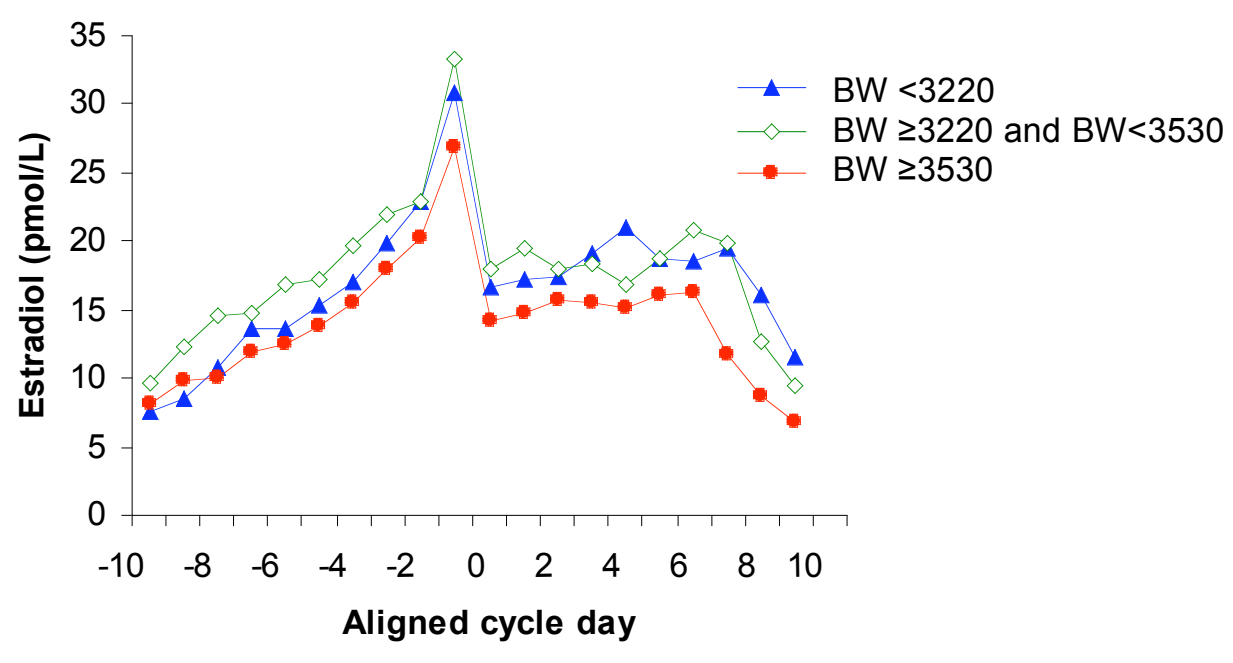

b

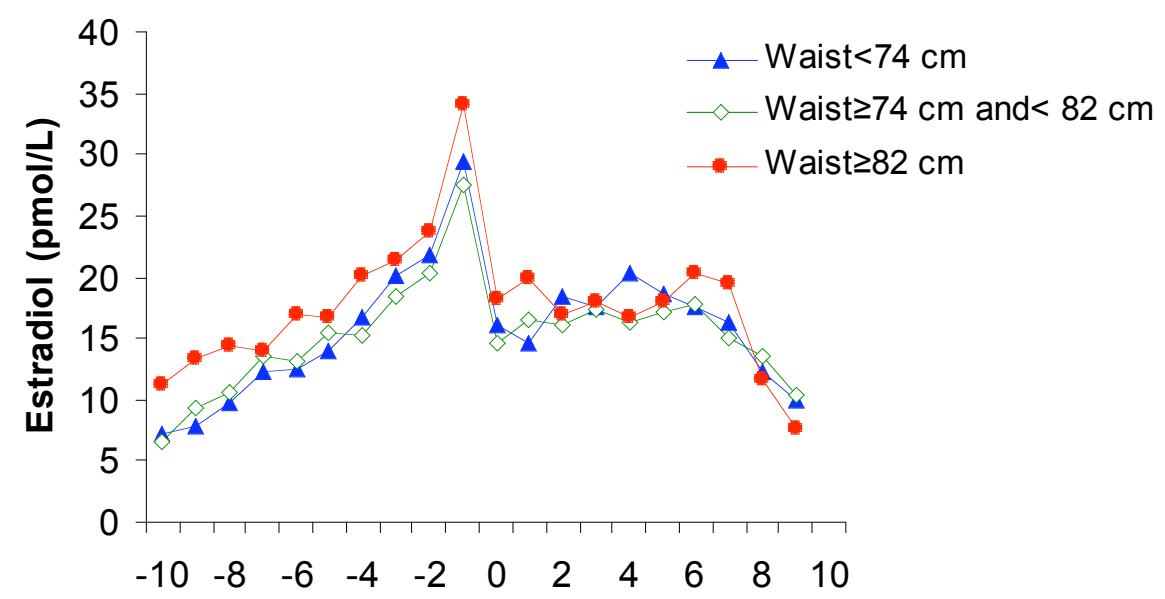

Aligned cycle day 


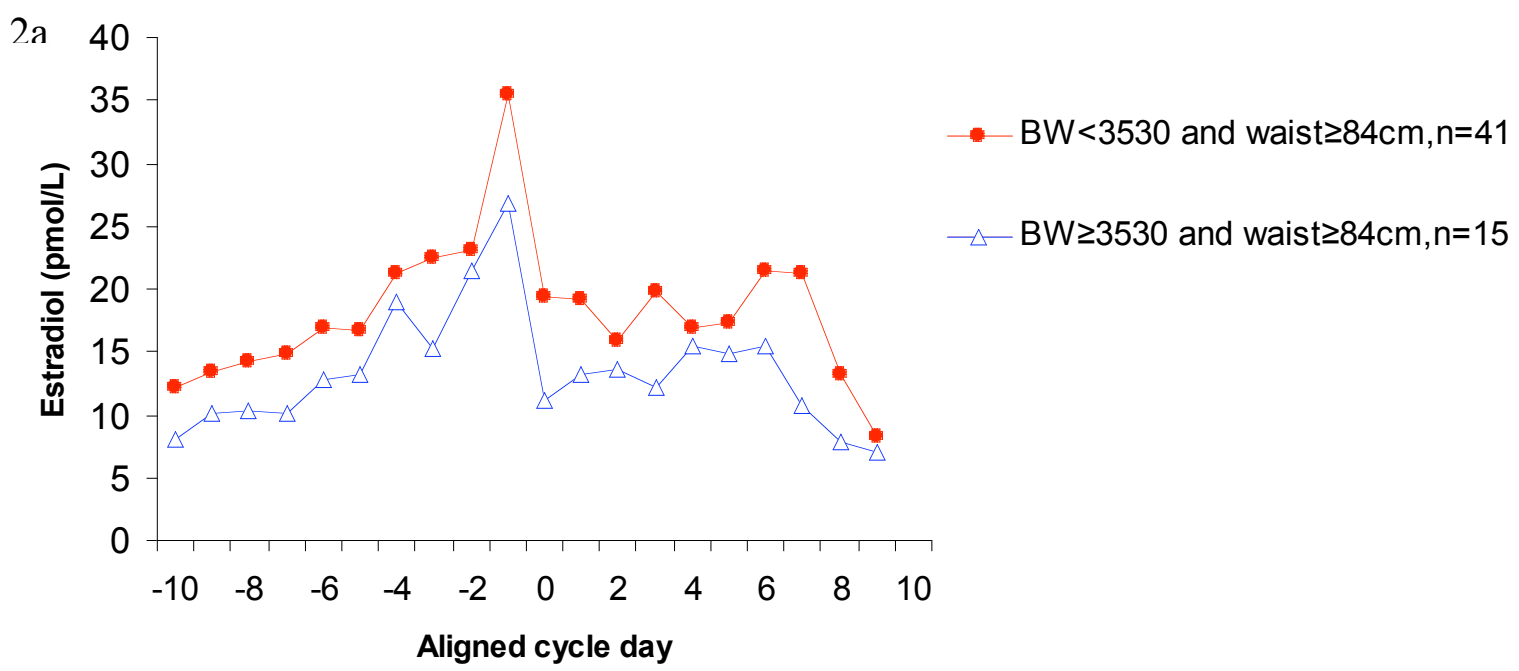

h

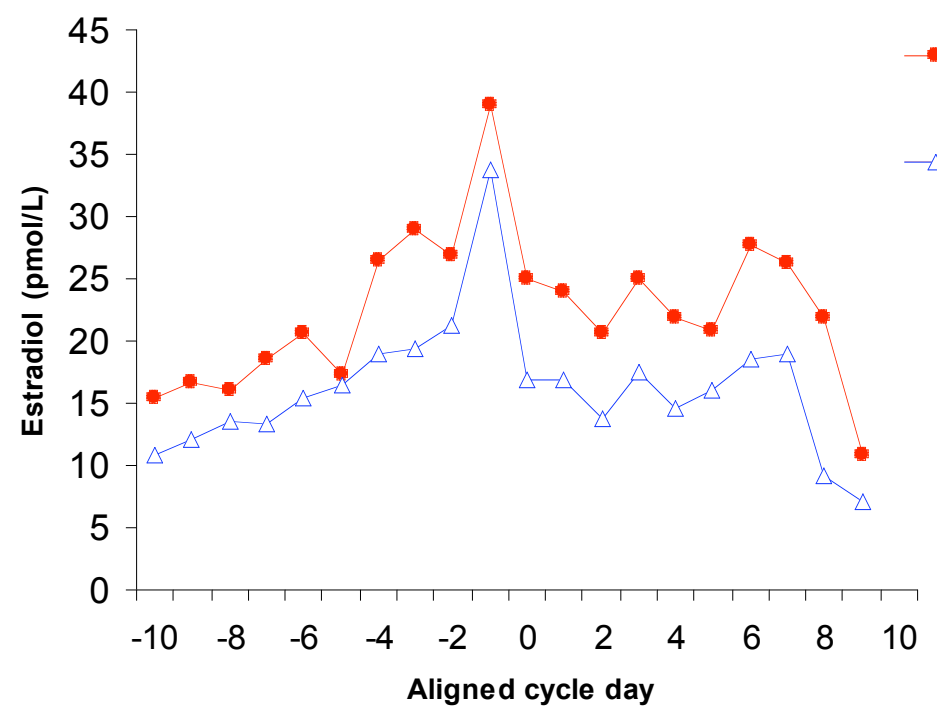

C

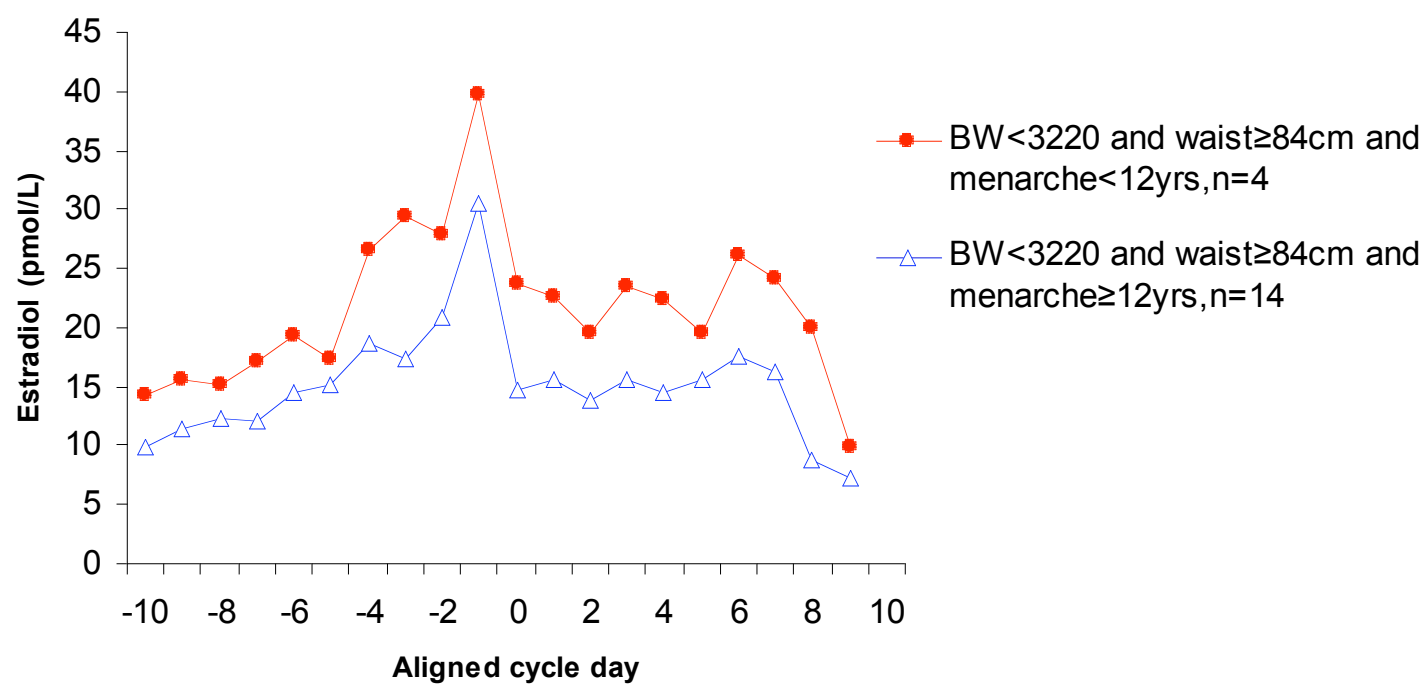


